\title{
Artigos
}

\section{Investigar la "otredad" en hospitales: desafíos en un estudio cualitativo sobre terapias no-convencionales en espacios de salud oficiales (Argentina)}

Investigating "alterity" in hospitals: the challenges of a qualitative study about nonconventional therapies in official healthcare spaces (Argentina) (abstract: p. 13)

Investigar a "alteridade" nos hospitais: desafios em um estudo qualitativo sobre terapias não convencionais em espaços de saúde oficial (Argentina) (resumo: p. 13)

\section{Mariana Bordes ${ }^{(a)}$}

<marianabordes@conicet.gov.ar> (a) CONICET, Centro Argentino de Etnología Americana. Avenida de Mayo, 1437, Piso 1, depto A, CP 1085. Buenos Aires, Argentina.

Este artículo analiza problemas asociados a un estudio cualitativo de orientación etnográfica que explora el fenómeno de las medicinas no-convencionales en contextos hospitalarios en Ciudad de Buenos Aires (Argentina). En diálogo con la literatura académica que explora los desafíos metodológicos, éticos y políticos de hacer etnografía en el ámbito sanitario, el artículo se divide en dos apartados: (1) uno que explora las dificultades asociadas al acceso; y (2) otro que analiza las dificultades que se desprenden del carácter sensible y marginal del tema a investigar. Se constata que las características del proceso de construcción del conocimiento no son extrañas a las relaciones de poder que atraviesan el campo a ser abordadas, y que las dinámicas sociales generadas en hospitales exigen respuestas negociadas y flexibles por parte del investigador.

Palabras clave: Hospitales. Medicinas no-convencionales. Desafíos etnográficos. 


\section{Introducción}

Este artículo constituye un ejercicio reflexivo que intenta (a la manera de una objetivación de la propia práctica de investigación social $\left.{ }^{1}\right)$ problematizar las dificultades/dilemas que emergieron en la etapa preliminar de un estudio sobre medicinas no-convencionales (desde ahora $\mathrm{MNC}$ ) en hospitales desde un enfoque sociológico cualitativo ${ }^{2}$ que incluyó la observación participante y entrevistas en profundidad como sus herramientas metodológicas principales. En particular, analiza los rasgos de "doble marginalidad” que signan la investigación, relativa no sólo al lugar subordinado del objeto de estudio (las MNC) en el campo de la atención sanitaria oficial, sino al del propio cientista social (cuyo saber ocupa una posición subalterna en la división del trabajo en el sector-salud). Consideramos que, entre otros conceptos elaborados desde la antropología y la sociología médicas, la noción de poder médico permite comprender la situación a analizar. Como propone Freidson ${ }^{3}$, la misma pone de relieve la capacidad de esta profesión de sostener una completa autonomía, no sólo para la regulación y control sobre la jurisdicción ocupacional que involucra al espacio social del arte de curar, sino a la hora de definir qué investigar -y quiénes pueden hacerlo- en su ámbito de injerencia. La afirmación de que este poder funda su legitimidad ante todo en una autoridad técnico-científica ${ }^{4}$ permite, a su vez, dotar de un referente conceptual a la postura jerárquica que garantiza la dominancia de la profesión médica por sobre otros actores sociales del campo de la salud, a partir de la definición de estatutos de cientificidad diferenciados (o nulos).

En lo que concierne a las MNC, la literatura sobre el tema coincide en señalar su surgimiento, en las últimas décadas, como un fuerte rival externo de la biomedicina: si bien se encuentran lejos de trastocar las estructuras de poder establecidas en sistemas sanitarios oficiales-, igualmente se registra un creciente consumo, particularmente entre grupos medios/altos urbanos, quienes adscriben cada vez más a sus postulados preventivos y holísticos 5 . La reciente estrategia incorporacionista adoptada desde el campo médico - que marca el pasaje del vocabulario que las define como formas de curanderismo al de "complementarias" ${ }_{\text {- }}$ surge entonces como una respuesta pragmática y defensiva, en vistas a salvaguardar su monopolio profesional y epistemológico. En términos profesionales, los grupos más conservadores reclaman la producción de datos estandarizados que demuestren la eficacia y seguridad de dichas terapias, redundando en una incorporación subordinada de aquellas formas terapéuticas consideradas más asimilables a la epistemología biologicista/materialista de la medicina ortodoxa (y una exclusión de las más esotéricas $)^{7}$. Al mismo tiempo, se registra la recuperación de estas disciplinas por parte de profesionales de la salud (sobre todo no-médicos) como una táctica para fortalecer su posición incorporando nuevas destrezas ${ }^{8,9}$. Lo que también puede ser leído bajo una lógica de expropiación que -según Conrad $^{10}$ - ya forma parte del normal accionar de la biomedicina.

El modo en que opera el poder médico no sólo expone las dificultades para referirnos a un pluralismo médico "pluralista" en términos estrictos ${ }^{11}$-lo que condujo a antropólogos médicos como Janzen ${ }^{12}$ a consignar su ineludible carácter asimétrico, al menos en ámbitos “oficiales" occidentales. También pone de relieve la necesidad de subrayar las limitaciones presentadas al cientista social cuando intenta adentrarse en espacios biomédicos. Retomando la tradición de las etnografías hospitalarias, diferentes autores han aportado a esta discusión. Por ejemplo, Van der Geest y Finkler ${ }^{13}$ afirman 
que ser cientista social e investigar en hospitales exige gestionar los procedimientos de monitoreo/control/exclusión que surgen de la desconfianza de autoridades y personal sanitario a la hora de conceder la entrada a estos profesionales outsiders. Asimismo, la adhesión a enfoques cualitativos y la propuesta de utilizar este tipo de técnicas radicaliza esta postura, generándose límites para su implementación: lo que va desde el pedido de estandarización de estas perspectivas flexibles ${ }^{14}$, hasta la imposición de restricciones para la participación ${ }^{15}$. Por último, en lo que respecta al abordaje de objetos de estudio marginados o "estigmatizados" en espacios médicos, se aduce la necesidad de redoblar los recaudos con los informantes, haciendo foco en el accionar ético del investigador e incluso redefiniendo aspectos esenciales de la investigación, con el fin de garantizar este $\operatorname{accionar}^{16}$.

El presente trabajo desarrolla dos apartados que problematizan estos desafíos, considerando las repercusiones actuales/potenciales que emergen en las diferentes etapas de la investigación: a) ¿con qué reacciones institucionalizadas se encuentra el cientista social al ingresar a este particular campo?, b) ¿Qué tensiones se vislumbran al recabar la información, sobre todo la asociada a prácticas terapéuticas culturalmente disruptivas para el campo médico?, c) ¿En qué sentido puede la difusión de resultados generar un daño a los informantes implicados?

Cabe destacar, en relación con los criterios éticos, que esta investigación fue avalada por la Comisión de Sociología, Demografía y Comunicación Social de CONICET. Asimismo, de acuerdo a las características de la investigación, me atuve al procedimiento del consentimiento informado ${ }^{17}$.

\section{Acceso: lograr el aval institucional}

La investigación se inició a partir de la aprobación, por parte del Consejo Nacional de Investigaciones Científicas y Técnicas (CONICET) del proyecto "Medicinas no-convencionales en ámbitos oficiales de salud. Un estudio de casos en torno de las dinámicas de inserción, práctica y legitimación de medicinas alternativas en marcos institucionales biomédicos (Ciudad de Buenos Aires)", centrado en analizar aspectos relativos a la coexistencia de esta medicina con la medicina ortodoxa y sus implicancias profesionales, culturales, institucionales. Teniendo como referencia otros estudios, sobre todo de la academia anglosajona, se puso el hincapié en analizar procesos de entrada/permanencia en hospitales por parte de terapeutas no-reconocidos en el ámbito de la salud oficial, en las dinámicas de cooperación/conflicto esbozadas en relación con el staff médico y paramédico, así como la morfología de su inscripción en la geografía hospitalaria. Consideramos apropiado elaborar una estrategia metodológica cualitativa retomando dos tradiciones de estudio: el estudio de casos - concebido como un "sistema delimitado en tiempo-espacio de actores, relaciones e instituciones sociales donde se busca ahondar en la particularidad del mismo en el marco de su complejidad"18 (p. 220) - y la etnografía. Si bien mi formación de base no es la antropología, consideré pertinente recuperar este enfoque en pos de las ventajas ofrecidas para el estudio de procesos emergentes, captando modos de interacción y lógicas para el establecimiento de definiciones en términos de la capacidad creativa de los agentes, sin olvidar constreñimientos estructurales/organizacionales ${ }^{19}$. Con la propuesta de particularizar al menos dos casos de práctica alternativa en servicios hospitalarios a seleccionar, comencé 
a conectar con grupos de voluntarios de amplia difusión en las redes sociales y, a través de ellos, entrar en contacto con profesionales de la salud. La estrategia para encarar el proyecto fue hacer un seguimiento de los itinerarios de dos grupos alternativos (practicantes de reiki y reflexología) evaluando las posibilidades de radicar la investigación en los espacios hospitalarios de trabajo de los mismos.

A pesar de la predisposición e interés por parte de terapeutas y profesionales de la salud que fui contactando, en esta etapa preliminar se manifestó una constatación realizada desde la literatura especializada: el ingreso al campo hospitalario expone al investigador cualitativo de las ciencias sociales a una serie de condicionamientos que exigen la adecuación de la estrategia inicial de investigación a las normas de la institución. Lo cual, en última instancia, tiende a un replanteo acerca de cómo debe llevarse a cabo la investigación. Esto asumió diferentes aristas.

En términos organizacionales, el desafío principal se puso de manifiesto a la hora de otorgar la formalidad requerida al estudio a través de la presentación del proyecto ante los respectivos comités de ética de la investigación de los hospitales recorridos $(\mathrm{n}=3)$. Esto se vinculó, no tanto las dificultades propias del proceso mismo de tramitación del pedido, sino mi carácter de "investigador externo", es decir, no formando parte del cuerpo de ocupaciones hospitalarias. Las implicancias de esta exterioridad pueden ilustrarse con un ejemplo en el que se observó que ni los datos de contacto del comité, ni las pautas operativas -expresadas generalmente bajo el formato de formularios estandarizados- se encontraban informatizados -lo que puede interpretarse en concordancia con el carácter relativamente reciente de las reglamentaciones que establecen la obligatoriedad de los CEI en hospitales de Argentina y las dificultades para ponerlos en funcionamiento en espacios públicos de salud ${ }^{20}$. El obstáculo de este "ser externo" se vinculó con el hecho de esforzarme por conseguir esta información en un espacio cuyas rutinas horarias y geografía me eran desconocidas. Asimismo, imponía una dependencia respecto del profesional de salud a cargo de la sala -quien debía hacer propia la investigación, asumiendo el compromiso de "encontrar" por los pasillos hospitalarios a algún miembro del consejo para transmitirme los datos de la próxima reunión del CEI. Para evitar la situación de exterioridad y otorgar legitimidad/plausibilidad al pedido, finalmente -con la mediación del punto de vista del director- se resuelve que el proyecto debía ser co-dirigido junto al jefe de sala. Lo que tuvo como consecuencia la pérdida de mi responsabilidad exclusiva respecto de la investigación en términos formales. Desde un punto de vista organizacional, también es posible mencionar el clima particular que rodeó este tipo de trámite en todos los casos. Algunos comentarios off the record de los informantes profesionales/terapeutas tendieron a desincentivar la presentación (¡no lo presentes!, como indicaba una obstétrica) argumentando el tiempo de espera -que, en Argentina, suele extenderse por meses, incluso años-; también se adujo la existencia de tensiones internas de índole personal/profesional, que podrían derivar en que algunos miembros del CEI "complicaran” el proceso.

En términos cognitivos, se observaron discrepancias respecto de los modelos culturales para hacer investigación sustentados por la ciencia médica y las ciencias sociales. El hecho de suscribir a un enfoque cualitativo desconcertó a médicos/otras profesiones de la salud lo que, según Hoeyer et al. ${ }^{21}$. se vincula con lo problemático de transmitir (en ámbitos médicos) objetivos que no adscriben ni a una hipótesis ni a una búsqueda de evidencias a través de procedimientos estandarizados ${ }^{22}$. También los enfoques visuali- 
zados como ajenos al paradigma biomédico aparecen en un rango de inferioridad, en lo que a la jerarquización de saberes respecta. Una nota de campo ilustra esta situación:

$\mathrm{X}$ (médico especialista) hizo una lectura rápida del proyecto, repitiendo "me interesa” en algunas ocasiones. Luego pregunta "¿con qué herramienta van a llevar a cabo el estudio?” Respondemos que desde un enfoque cualitativo, realizaríamos entrevistas en profundidad y observaciones. El doctor desestima con un gesto la propuesta, responde que existen diferentes tests que deberíamos retomar para medir la calidad de vida. Para pasar por el comité de ética y el departamento de Docencia e Investigación son lo más adecuado. Le comentamos sobre nuestra mirada y las ventajas que ofrece. Pero enfatiza que se trata de tests desarrollados internacionalmente y que nadie discute, y que además aseguran la posibilidad de publicar. También propone trabajar con un grupo de control. (Nota de campo, 7 de marzo 2017)

Si la respuesta del profesional puede interpretarse en virtud de mi carácter de investigador externo y de cientista social que adscribe a una orientación cualitativa, cabe destacar que distinta fue la situación respecto de las tensiones asociadas a la especificidad del objeto de estudio (MNC), en tanto los mecanismos de rechazo/control se expresaron en dificultades experimentadas, no por mí, sino por los profesionales de la salud contactados. Esto se hizo evidente en dos escenas protagonizadas por una médica y una terapista ocupacional, quienes contaban con largas trayectorias de pugnas internas para integrar este tipo de ofertas heterodoxas en sus prácticas hospitalarias. La diferencia entre ambas fue que en la segunda, las tensiones previas se recrudecen al visibilizarse la presente propuesta de investigación.

Yo comento que después del primer período de observaciones quisiera formalizar mi trabajo presentando el proyecto al CEI del hospital. La doctora responde afirmativamente, y enfáticamente agrega 'pero no me quiero pelear'. Ante mi sorpresa, aclara que ya ha enfrentado inconvenientes al realizar sus propias propuestas de investigación -incluyendo terapéuticas 'alternativas'- que quedaron truncas por los eternos pedidos de revisión y virtual 'cajoneo'. Se trata de una instancia que deberé enfrentar sola, si bien se ofrece a facilitar la firma del jefe de la sala para iniciar el trámite. (Nota de campo, 11 de febrero 2016)

Lo primero que hizo X cuando llegué fue relatarme, angustiada, lo incómodo que había sido ir al comité de ética del hospital. Me decía que al entrar al recinto todos le hicieron una especie de "vacío", ella trataba de hablarles y el grupo hacía caso omiso. En una ocasión anterior había hablado con un miembro del mismo, relatándole que iba a colaborar con una investigadora de CONICET interesada en la terapia que se ofertaba en su piso (reiki). Supuso que esta persona les había contado a todos. Me comenta que, perdiendo la paciencia, logra obtener el formulario [necesario para la presentación], mientras agita el borrador del proyecto aclamando a viva voz: "se trata de un proyecto sobre calidad de vida, nada más". (Nota de campo, 7 de noviembre 2016) 
Castro $^{23}$ señala que una de las implicancias del ejercicio del poder médico es "la capacidad de imponer los propios esquemas de percepción y apreciación de la realidad (...), así como las reglas de operación y evaluación de los diversos actores del campo sanitario” (p. 135). Este rasgo, ligado al goce de autonomía en el control de su propia materia de conocimiento, la habilita a delimitar las fronteras de lo que es legítimo o no, no sólo para la práctica médica, sino para todas las otras ocupaciones que se desenvuelvan en el ámbito sanitario (entre las cuales contamos a las ciencias sociales) ${ }^{(b)}$. Sin embargo, los criterios últimos a partir de los cuales estas fronteras se definen, no dependen tanto de cuestiones científicas, sino que emergen como un efecto directo de una estructura de poder jerarquizada que, para el caso que nos ocupa, reproduce la descalificación de saberes "otros" (sean MNC o ciencias sociales).

En consonancia con esta lectura, la literatura sobre el tema esgrime el concepto de gatekeeper en pos de interpretar las dificultades para el acceso a ámbitos hospitalarios. Éste hace referencia a aquellos actores sociales que, en virtud de su autoridad cognitiva y sus recursos materiales/simbólicos, definen las fronteras de un determinado dominio -en este caso, el médico ${ }^{27}$ - según una lógica jurisdiccional que demarca un adentro y un afuera. En hospitales, esta delimitación supone la no-admisión (o su relegamiento a un lugar subordinado/deslegitimado) de aquellos actores definidos bajo la segunda clasificación. ¿Qué ocurre con aquellos profesionales ortodoxos que insisten en valorizar estas prácticas? Como observamos, son objeto de sanciones informales por parte de aquellos colegas que adoptan las posiciones más conservadoras sobre la temática. Lo que genera un obstáculo suplementario para los investigadores jerárquicamente rezagados que dependen de "apoyos" médicos y/o paramédicos para avanzar con tareas de investigación.

\section{Producción de datos y difusión: abordar un objeto de estudio marginado}

\section{El riesgo de exotizar la diferencia epistemológica: qué incluir en el recorte}

Uno de los tópicos recurrentes en la literatura sobre la reflexividad del investigador social que trabaja en hospitales es el del rol del etnógrafo dentro del campo y sus implicancias limitantes. Entre las posibilidades, la más habitual es la de acoplarse al staff médico; "devenir" en paciente; o asumir el rol de visitante ${ }^{13}$. Wind ${ }^{15}$, por su parte, problematiza el rol del "investigador" propiamente dicho, el que reconoce límites que afectan los alcances de la observación participante -como, por ejemplo, presenciar sólo aquellas actividades habilitadas para ser presenciadas (excluyendo áreas restringidas, pacientes internados, consultas médicas).

Este punto cobra relevancia para reflexionar sobre esta investigación ya que, en tanto investigadora de CONICET, este rol fue el asumido. Mi interés en las MNC -y dado que en su mayoría se practicaban en un espacio/tiempo destinado a esa tarea, sin el involucramiento directo de los profesionales de salud-implicó que la participación se planteara acompañando a los terapeutas "alternativos": charlas con otros actores (médicos/enfermeras/voluntarios), reuniones de grupo y sesiones terapéuticas. En el marco de estas dos últimas instancias se impuso un interrogante (práctico y a la vez éti-

\begin{abstract}
(b) La autonomía médica y el consecuente lugar dominante por sobre otras ocupaciones fue logrado no sólo en virtud de sus avances tecnológicos sino a partir de un intrincado proceso histórico de organización corporativa y de obtención de apoyo estatal $^{4}$. La construcción de un campo de la salud oficial circunscripto a las prácticas médicas ortodoxas supuso una serie de procesos de exclusión pero también de incorporación/legitimación de diferentes saberes a través de estrategias cognitivas pero también retóricas (de nuevos estratos ocupacionales: enfermeras y obstetras ${ }^{24}$; de especialidades subalternas como la kinesiología ${ }^{25}$ ). En la actualidad, las MNC replican estos procesos con rasgos propios (ver, p.e, ${ }^{26}$ ).
\end{abstract}


co) acerca de cómo actuar, qué hacer con la información recabada. Como ha indicado Maluf ${ }^{28}$ las terapias alternativas - particularmente en sus variantes más espiritualizadassuponen el despliegue de movimientos, gestualidades y posturas plenas de simbolismos y estructuras rituales; el espacio suele ser calificado con aromatizantes y música; incluso se incluye la vocalización de recitaciones u oraciones. El siguiente fragmento de mis notas de campo constituye una muestra de lo señalado:

En un momento el coordinador pregunta "¿Alguien puso símbolos en la habitación?”, los terapeutas responden que no, y X comienza a dibujar en el aire "signos", con el dedo índice del brazo derecho levantado, lo que repite cuatro veces, posicionándose en los cuatro puntos cardinales (adelante, derecha, izquierda y atrás). X pregunta también quién trajo la cartulina donde se observan dos símbolos violetas del yin y el yang. (Nota de campo, 17 de setiembre 2015)

¿En qué medida y desde qué enfoque es posible incluir este tipo de información como dato? Considerando que el marco de estos saberes/prácticas es un hospital, es decir, una institución social donde los profesionales de la salud fundan la legitimidad de sus prácticas en una autoridad derivada del carácter técnico-científico del conocimiento que vehiculizan: ¿Qué reacciones negativas puede ocasionar este abordaje, tanto para el cientista social como para los informantes implicados?

Este interrogante adquiere fuerza a partir del trabajo de campo preliminar, en el cual evaluamos que existía una probabilidad de interpretación con connotaciones negativas en relación con creencias y/o prácticas susceptibles de ser definidas como "irracionales" en el seno de esta institución social. Teniendo en cuenta la distancia cultural involucrada respecto de las prácticas clínicas de la biomedicina, estas prácticas aparecen como disruptivas, sin dudas. Esto es consignado por Keshet ${ }^{7}$, quien especifica que las posturas ortodoxas del campo biomédico no plantean solamente el argumento de la ineficacia, el carácter ilógico de sus tratamientos, situándolas por fuera de las posibilidades de corroboración científica. Todo lo que sea leído como irracional en estas terapias, deviene en "potencialmente amenazador, corruptor o destructor del conocimiento médico” (p. 141). Estas referencias despectivas respecto de las MNC se registraron en las narrativas de los informantes, quienes relataron escenas específicas: los dichos de una autoridad que, si bien avalaba la implementación terapéutica no-convencional en uno de los servicios enfatiza que "por favor no se enciendan ni velas ni nada raro" (cuando la práctica en cuestión consiste en una imposición de manos). Comentarios acerca de un grupo de colegas que, al tomar conocimiento de la oferta alternativa, solicitan una reunión con el director del hospital, alegando que "el hospital no es lugar para prácticas de espiritismo". Una obstétrica que afirma "a mí me querían quemar en la hoguera”, refiriendo una lógica inquisidora en el seno de la comunidad científica contra todo aquello considerado esotérico.

En la medida en que fueron apareciendo, estos relatos breves -entre otros- se constituyeron en indicios de una visión particular sobre estas terapias, articulada con un imaginario social que podríamos denominar como exotizante. Así, al ubicarlas por fuera del paradigma biomédico, se registra un mecanismo retórico al interior del hospital que tiende a situarlas en un lugar de fronteras difusas, definiéndolas como una actividad desviada respecto no sólo de un conjunto de normas que regirían la práctica médica 
científica, sino incluso de las religiones institucionalizadas -como enfatiza el término "espiritismo", esgrimido aquí peyorativamente. También registramos otra postura más benevolente, como cuando un médico nos explica "el espacio que más personas convoca el hospital es la iglesia”. De este modo, estas terapias se sitúan en el ámbito de la creencia, asociada al relativismo cultural y al efecto placebo ${ }^{29}$. Definidas así, asumen un estatuto de inocuidad respecto de sus efectos empíricamente comprobables, a la vez que se contemplan posibles beneficios a nivel mental-espiritual.

El tópico de la marginación de las MNC vía su exotización expone, para el cientista social, no sólo los mecanismos retóricos que operan desprestigiando a las terapias competidoras al interior de la institución hospitalaria, sino también las tensiones existentes entre los conflictos inherentes al campo de estudio y las exigencias disciplinares. Para el caso de la presente investigación, esta tensiones contraponen las potenciales implicancias adversas si la información "controversial” recabada fuera retomada por una audiencia contraria a las prácticas no-biomédicas (suponiendo el eventual descrédito que podría acarrear para grupos terapéuticos deseosos de ser aceptados en el ámbito oficial) y el genuino interés del investigador por construir/analizar datos para la divulgación de resultados (particularmente cuando estos datos constituyen gran parte de la información compilada, como en mi caso). No existen fórmulas a seguir ni soluciones predefinidas en lo que a la literatura respecta. Anspach y Mizrachi ${ }^{30}$ consignan el peligro de caer en un "dilema de discreción" si, al evitar aquellos asuntos que pudieran perjudicar las relaciones en el campo, se sacrificaran los estándares de la buena etnografía. Smith $^{31}$ alerta acerca de la situación contraria: el riesgo de retomar la información que las personas suministraron de buen grado pero que eventualmente pudiera devenir en dañina. El problema con las MNC es que constituyen para la propia disciplina sociológica una temática que corre el riesgo de exotizarse si se privilegian aquellos recortes del fenómeno que enfaticen su carácter de otredad ${ }^{32}$. En el ámbito hospitalario, este riesgo adquiere una nueva arista, ya que la descripción de los aspectos menos asimilables a la mirada biomédica, podría terminar reificando estas terapias en tanto "pseudociencias"; contribuyendo a recrudecer las actitudes de rechazo más ortodoxas a nivel local.

\section{El riesgo de la sanción/expulsión: difundir los resultados en un contexto de ocultamiento}

Este subapartado se encuentra vinculado con el planteo recién desarrollado, pero operando otro énfasis. Mientras que el subapartado anterior aborda los desafíos etnográficos asociados a los rasgos estigmatizados que -bajo la forma de la retórica del exotismo- asumen las MNC en los ámbitos hospitalarios explorados, aquí se expone cómo las críticas/rechazos se vuelcan hacia los actores sociales que encarnan a estas opciones terapéuticas, y las implicancias que este hecho define en términos prácticos/éticos.

Una de las primeras constataciones al ingresar al campo de estudio fueron las prácticas/estrategias adaptativas desarrolladas por terapeutas -y por parte de profesionales de la salud que esgrimen estas opciones-, que emergen como modos efectivos para negociar la permanencia en un espacio donde rigen mecanismos de control/sanción de aquellos comportamientos que se alejan de las normas institucionales. Según lo recabado, cabe mencionar tres tipos de estrategias que se corresponden con esta lógica. 
Primero, una estrategia de mímesis - que la literatura define como isomorfismo institucional- que supone el uso de chaquetas/ambos, el registro de las historias clínicas en fichas, la organización de jornadas de capacitación con profesionales de la salud. Como indican Mizrachi et al. ${ }^{29}$ a través de este tipo de prácticas, los alternativos se someten a las estructuras de poder existentes evitando "marcar" un territorio distinto al interior del campo biomédico, con el objetivo de mitigar todo efecto disruptivo en el hospital. Segundo, se identificaron estrategias de "minimización" del propio trabajo, con la intención de des-jerarquizar la propuesta terapéutica y sus posibles efectos, en un acatamiento del lugar marginal atribuido institucionalmente ("te voy a hacer un masajito que te va a relajar") ubicándose por fuera de todo conflicto asociado al ejercicio ilegal de la medicina. Por último, se distinguió una estrategia de ocultamiento, expresada en un continuum de esfuerzos cotidianos tendientes a regular la visibilidad de las tareas realizadas (o, incluso, de la propia presencia). En algunos casos, se destacó una construcción del espacio terapéutico en términos de enclave. En efecto, una vez que la terapia ha sido aceptada en determinado servicio, una opción para la atención suele ser que se les otorgue a los terapeutas la disponibilidad de un recinto para la ejecución de la práctica, en un horario y días determinados. Este espacio tiende a constituirse en un "bunker alternativo", con sus propias marcaciones identitarias (símbolos, imágenes, colores) y su realidad terapéutica. Este hermetismo se refuerza si la realidad del hospital circundante es leída como hostil: gestos de fastidio del personal de salas contiguas, indiferencia o burla del equipo de salud, interrupción de sesiones. Como respuesta, la práctica del silencio, el cierre estricto de puertas para custodiar aquello que sucede en el interior, evitar encender aromatizantes pasibles de ser percibidos desde el exterior, resultan habituales. El costo de la exposición pública, contemplando la extrema informalidad ocupacional que caracteriza a los terapeutas no-convencionales (sin remuneración ni contrato), puede repercutir directamente en la pérdida del espacio ganado. Como indica una terapeuta, relatando su experiencia en un hospital en donde las sesiones justamente no se realizaban en recintos cerrados:

En el [nombre de hospital] todavía estábamos más en silencio porque teníamos que andar por los pasillos, íbamos al sector de Internación, entrábamos y salíamos de las habitaciones [...]. La persona de seguridad nos vio salir cuando nos reuníamos todos en la sala de espera para terminar... nos preguntó si teníamos permiso. Le dijimos sí, de [nombre de director], de [jefes de servicio]... Él habrá querido resguardar su trabajo y dio aviso de ese tema y ahí [al director] dijo veamos si la junta médica lo acepta. Y la junta lo aceptó pero volviendo al director, no nos permitió ir más [...] (Entrevista a terapeuta)

Por su parte, los profesionales de la salud que adscriben a saberes "alternativos" monitorean y regulan a través de estas estrategias frente a quiénes develar su práctica. La exposición a situaciones de sanción y/o desprestigio a partir de la visibilización de la práctica vienen morigeradas, empero, por el estatus profesional (médico/no-médico) la posición institucional (antigüedad en el cargo, especialidad ${ }^{(\mathrm{c})}$ ) y por el grado de exposición ante otros.

\footnotetext{
(c) En distintas áreas de atención-Oncología/ Cuidados Paliativos- los profesionales se encuentran más habilitados a vehiculizar perspectivas (y técnicas) que conciban al paciente como un ser "integral". Por ende, cuentan con más "libertad" para incluir MNC.
} 
Me ha pasado que me miran raro, dicen "qué se van a fumar" y hacen comentarios irónicos pero en mi caso no lo boicotean [...] al tener más años en la institución te consideran más par, ya te van conociendo. Entonces no es una cosa de cuestionarte todo, no te sentís tan amenazado. (Entrevista a obstétrica que aplica reiki/técnicas de relajación)

Nadie sabe bien lo que hace un TO [terapista ocupacional], entonces yo trabajo según mi experiencia y las herramientas que fui sumando. Ellos [equipo de salud mental] me mandan gente y ven que lo que hago funciona. Nadie me pregunta qué hago exactamente [...] me he formado bastante en psicoanálisis y encuentro muchas similitudes con las técnicas que aplico. Según el público, veo de pasar al registro del psicoanálisis, voy "modulando el dial”. (Entrevista a terapista ocupacional que instrumenta técnicas de visualización/gemoterapia/astrología)

Esta postura de recaudo devino también en una exigencia por mantener ciertas charlas informales off the record, debido a temores de reprimendas y/o de poner en riesgo el trabajo, lo que observamos en el caso de profesionales de ingreso más reciente en el cargo.

Lo antedicho expone el modo en que la demarcación entre un "adentro" y un "afuera" del campo médico definido como legítimo desde una lectura ortodoxa, se expresa en reacciones negativas hacia los individuos que practican MNC en hospitales. Podríamos interrogarnos aquí cómo es posible dotar de visibilidad a estas prácticas -y hasta qué punto- en articulación con las propias estrategias de evitación del daño desarrolladas por los informantes. Desde el campo de las ciencias sociales, el consentimiento informado y el recurso de la anonimización es la vía por antonomasia para gestionar tensiones emergentes. Por ejemplo, de manera preliminar decidí retirar no sólo los nombres sino las disciplinas específicas en las que se posicionan ("terapeuta" en lugar de "reflexólogo" o reikista) o cambiar el nombre de las especialidades, cuando pueden ser consideradas equivalentes (una profesión no-médica por otra) y el género, como una manera de no ofrecer marcaciones identitarias directas. Empero, seguiría incluyendo los rasgos del servicio/espacio hospitalario de referencia (sin mencionar el nombre del hospital). No obstante, las posibilidades de identificación no son nunca anuladas del todo, como indican Saunders et al. ${ }^{33}$, y esto se constata en un campo de estudio en que los actores implicados suelen ser reconocidos en su medio social, al constituir un circuito terapéutico reducido. Es por esto que adquiere relevancia la posibilidad de negociar la información pasible de ser incluida y cuál es preciso retirar del estudio, desde una lógica intersubjetiva de preservación de la confidencialidad, en que investigador e informantes evalúan juntos las implicancias de los datos a difundir ${ }^{34}$. Esto implica evaluar la situación particular de cada informante, el contexto en el que trabaja y su posición en la estructura profesional hospitalaria, para determinar el grado de marginalidad y riesgo de daño, que no es equiparable en todos los casos relevados.

\section{Conclusiones}

El objetivo del trabajo fue analizar los desafíos relativos al proceso de construcción, en tanto objeto de estudio sociológico, del fenómeno asociado a la presencia de MNC 
en hospitales. Me enfoqué en especificar los aspectos más problemáticos identificados relativos al acceso, la producción de datos y la potencial difusión de los mismos, en un ejercicio reflexivo acerca de las condiciones de producción de conocimiento, que se constituye cada vez más en una exigencia de la práctica académica de las ciencias sociales $^{1}$. De acuerdo con los antecedentes sobre el análisis reflexivo en etnografía, estructuré las tensiones avizoradas considerando, primero, los desafíos asociados al modo en que la institución social hospitalaria acciona y reacciona frente al cientista social que vehiculiza enfoques cualitativos (externo, además, al campo sanitario). Así, se explicitó que el acceso supone como condición previa una aceptación de los modos de investigar de la institución biomédica, de acuerdo con los mecanismos asociados a la lógica del gatekeeper. En segundo lugar, especifiqué el desafío de abordar un objeto de estudio definido como marginal, y el modo en que este rasgo del fenómeno exige poner el foco en la protección de los informantes como responsabilidad del investigador. Esto supuso enfrentar el dilema acerca de cómo y hasta qué punto incluir información sensible sin afectar los estándares de la calidad de la investigación; sobre todo cuando la visibilización de ciertas prácticas pone de manifiesto su carácter culturalmente disruptivo, teniendo dos consecuencias potenciales: a) contribuir a reforzar un mecanismo retórico de control/exclusión vigente en el campo hospitalario sobre la base de la exotización de las MNC, en particular, de las más espiritualizadas (como el reiki) y b) contribuir a reforzar mecanismos organizacionales de sanción (desprestigio/reprimenda/expulsión) sobre terapeutas y profesionales.

La temática abordada pone de manifiesto el hecho de que la producción de conocimiento no es ajena a las relaciones de poder que atraviesan el campo a abordar. La constante negociación ante los constreñimientos impuestos desde las dinámicas organizacionales (considerando que el hospital constituye un espacio social particularmente restrictivo) exige que el cientista social se vea compelido a explicitar las condiciones bajo las cuales le resulta posible definir su objeto de estudio, lo que garantiza una comprensión más cabal de sus decisiones investigativas.

\section{Derechos de autor}

Este artículo está bajo la Licencia Internacional Creative Commons 4.0, tipo BY (https://creativecommons.org/licenses/by/4.0/deed.es).

\section{Referencias}

1. Bourdieu P, Wacquant L. Una invitación a la sociología reflexiva. Buenos Aires: Siglo XXI Editores; 2015.

2. Minayo MC. La artesanía de la investigación cualitativa. Lanús: Lugar; 2009.

3. Freidson E. Profession of medicine: a study of the sociology of applied knowledge. Chicago: University of Chicago Press; 1988.

4. Starr P. The social transformation of American medicine. New York: Basic Books; 1982. 
5. Astin JA. Why patients use alternative medicine: results of a national study. JAMA. 1998; 279(19):1548-53.

6. Winnick T. From quackery to "complementary" medicine: the American medical profession confronts alternative therapies. Soc Probl. 2005; 52(1):38-61.

7. Keshet Y. The untenable boundaries of biomedical knowledge: epistemologies and rhetoric strategies in the debate over evaluating complementary and alternative medicine. Health. 2009; 13(2):131-55.

8. Cant S, Watts P, Ruston A. Negotiating competency, professionalism and risk: the integration of complementary and alternative medicine by nurses and midwives in NHS hospitals. Soc Sci Med. 2011; 72(4):529-36.

9. Tovey P, Adams J. Nostalgic and nostophobic referencing and the authentication of nurses' use of complementary therapies. Soc Sci Med. 2003; 56(7):1469-80.

10. Conrad P. The medicalization of society: on the transformation of human conditions into treatable disorders. Baltimore: JHU Press; 2008.

11. Singer M. Reinventing medical anthropology: toward a critical realignment. Soc Sci Med. 1990; 30(2):179-87.

12. Janzen J. The social fabric of health: an introduction to medical anthropology. Boston: MCGraw-Hill; 2002.

13. Van der Geest S, Finkler K. Hospital ethnography: introduction. Soc Sci Med. 2004; 59(10):1995-2001.

14. Gotlib L. Ethics policy as audit in Canadian clinical settings: exiling the ethnographic method. Qual Res. 2008; 8(4):499-514.

15. Wind G. Negotiated interactive observation: doing fieldwork in hospital settings. Med Anthropol. 2008; 15(2):79-89.

16. Inhorn M. Privacy, privatization and politics of patronage: ethnographic challenges to penetrating the secret world of Middle Eastern hospital-based in vitro fertilization. Soc Sci Med. 2004; 59(10):2095-108.

17. Lipson JG. Ethical issues in ethnography. In: Morse J, editor. Critical issues in qualitative research methods. Thousand Oaks: Sage; 1994. p. 333-55.

18. Neiman G, Quaranta G. Los estudios de caso en la investigación sociológica. In: Gialdino IV, cordenadora. Estrategias de investigación cualitativa. Madrid: Gedisa; 2006. p. 213-37.

19. Charmaz K, Olesen V. Ethnographic research in medical sociology: its foci and distinctive contributions. Sociol Methods Res. 1997; 25(4):452-94.

20. Sabio M, Bortz J. Estructura y funcionamiento de los comités de ética en investigación de ciudad de Buenos Aires y Gran Buenos Aires. Salud Colect. 2015; 11(2):247-60.

21. Hoeyer K, Dahlager L, Lynöe N. Conflicting notions of research ethics: the mutually challenging traditions of social scientists and medical researchers. Soc Sci Med. 2005; 61(8):1741-9.

22. Barry $\mathrm{C}$. The role of evidence in alternative medicine: contrasting biomedical and anthropological approaches. Soc Sci Med. 2006; 62(11):2646-57.

23. Castro R. Teoría social y salud. Lanús: Lugar Editorial; 2011.

24. Foley L, Christopher AF. Medicine as discursive resource: legitimation in the work narratives of midwives. Sociol Health Illn. 2003; 25(2):165-84. 
25. Øvretveit J. Medical dominance and the development of professional autonomy in physiotherapy. Sociol Health Illn. 1985; 7(1):76-93.

26. Lambert H. Medical pluralism and medical marginality: bone doctors and the selective legitimation of therapeutic expertise in India. Soc Sci Med. 2012; 74(7):1029-36.

27. Shuval JT, Mizrachi N, Smetannikov E. Entering the well-guarded fortress: alternative practitioners in hospital settings. Soc Sci Med. 2002; 55(10):1745-55.

28. Maluf SW. Mitos coletivos, narrativas pessoais: cura ritual, trabalho terapêutico e emergência do sujeito nas culturas da "Nova Era”. Mana. 2005; 11(2):499-528.

29. Mizrachi N, Shuval J, Gross S. Boundary at work: alternative medicine in biomedical settings. Sociol Health Illn. 2005; 27(1):20-43.

30. Anspach RR, Mizrachi N. The field worker's fields: ethics, ethnography and medical sociology. Sociol Health Illn. 2006; 28(6):713-31.

31. Smith L. How ethical is ethical research? Recruiting marginalized, vulnerable groups into health services research. J Adv Nurs. 2008; 62(2):248-57.

32. Sharma U. Contextualizing alternative medicine. Anthropol Today. 1993; 9(4):15-8.

33. Saunders B, Kitzinger J, Kitzinger C. Anonymising interview data: challenges and compromise in practice. Qual Res. 2015; 15(5):616-32.

34. Wiles R, Charles V, Crow G, Heath S. Researching-researchers: lessons for research ethics. Qual Res. 2006; 6(3):283-99.

The present article addresses the issues faced in a qualitative ethnographic study that explores the phenomenon of nonconventional medicine in hospital environments in Buenos Aires (Argentina). While dialoguing with the academic literature that explores the methodological, ethical and political challenges of ethnography in the health field, the article is divided into two sections: 1) an exploration of the difficulties associated with access; and 2) an analysis of the barriers that result from the sensitive and marginal nature of the investigated phenomenon. The characteristics of the knowledge construction process are not unassociated from the power relations that involve the investigated field, and that the social dynamics generated in hospitals requires negotiated and flexible responses from the researcher.

Keywords: Hospitals. Nonconventional medicine. Ethnographic challenges.

Este artigo discute os problemas encontrados em um estudo qualitativo de orientação etnográfica que explora o fenômeno das medicinas não-convencionais em ambientes hospitalares, em Buenos Aires (Argentina). Em diálogo com a literatura acadêmica que explora os desafios metodológicos, éticos e políticos da etnografia no campo da saúde, o artigo é dividido em duas seções: (1) uma que explora as dificuldades associadas ao acesso; e (2) outro que analise as dificuldades que surgem da natureza sensível e marginal do fenômeno a ser investigado. Observa-se o fato de que as características do processo de construção do conhecimento não são estranhas às relações de poder que atravessam o campo a serem abordadas, e que a dinâmica social gerada nos hospitais exige respostas negociadas e flexíveis pelo pesquisador.

Palavras-chave: Hospitais. Medicinas não-convencionais. Desafios etnográficos. 